\section{SCIENCE CHINA \\ Physics, Mechanics \& Astronomy}

-Editorial•

Editor's Focus
June 2020 Vol. 63 No. 6: 267061

https://doi.org/10.1007/s11433-020-1534-5

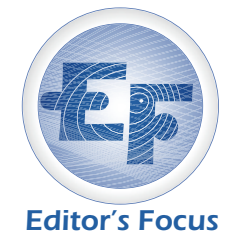

\title{
Topological insulators induced by disorder and non-Hermiticity
}

Searching for topological states of matter in real materials or engineered systems has been a fundamental theme in condensed matter physics in the past decade [1]. It is known that the topological insulators are robust against certain disorders, but they usually become trivial under strong disorders due to the Anderson localization. In the year 2009, it was theoretically found that a topological insulator can be surprisingly driven from a trivial phase by disorders, which is called the topological Anderson insulator [2]. Although the topological Anderson insulator has been studied in various theoretical models [3-5], it was not until 2018 that its experimental observation was reported in two different artificial systems [6,7], i.e., the one-dimensional cold atomic wires and two-dimensional photonic waveguide arrays.

To date, the study of topological states mainly focuses on Hermitian systems [1-7] that naturally exist in ordinary condensedmatter materials. Inspired by recent advances in engineering non-Hermiticity in artificial systems, more and more efforts are made to explore non-Hermitian topological phases [8-10]. The interplay among topology, disorder, and non-Hermiticity may induce unknown physical phenomena. In a recent study, Zhang et al. [11] from the South China Normal University and Nanjing University explored the physics of the interplay and found some perceptive results. Explicitly, by focusing on the onedimensional chiral chains with disordered and nonreciprocal hopping terms, they showed that the non-Hermiticity could enhance the topological phase against disorders, and subsequently uncovered a non-Hermitian topological Anderson insulator induced by the combination of moderate non-Hermiticity and disorders. The non-Hermitian disordered system was revealed to have the unique non-monotonous localization behavior and intrinsic topological nature of Anderson transitions. More recently, the non-Hermitian topological Anderson insulators induced by purely non-Hermitian disorders were revealed in a complementary investigation [12].

The proposed non-Hermitian topological Anderson insulators represent a new kind of topological state, and the present work may thus stimulate further studies of the rich and colorful phenomena induced by the interplay among topology, disorder, and non-Hermiticity. Intriguingly, such topological states may be realized experimentally in the near future with ultracold atoms, photonic crystals, or electronic circuits, since tunable disorders and non-Hermiticity have been respectively engineered in these artificial systems and combining these ingredients is likely in experiments. This work may push forward the advancement in the exploration of exotic non-Hermitian topological phases and has a broad interest in the fields of condensed matter physics, nonHermitian quantum physics, and artificial topological systems.

XinCheng Xie

School of Physics, Peking University, Beijing 100871, China

1 D. W. Zhang, Y. Q. Zhu, Y. X. Zhao, H. Yan, and S. L. Zhu, Adv. Phys. 67, 253 (2018), arXiv: 1810.09228.

2 J. Li, R. L. Chu, J. K. Jain, and S. Q. Shen, Phys. Rev. Lett. 102, 136806 (2009), arXiv: 0811.3045.

3 H. Jiang, L. Wang, Q. F. Sun, and X. C. Xie, Phys. Rev. B 80, 165316 (2009), arXiv: 0905.4550.

4 C. W. Groth, M. Wimmer, A. R. Akhmerov, J. Tworzydło, and C. W. J. Beenakker, Phys. Rev. Lett. 103, 196805 (2009), arXiv: 0908.0881.

5 B. Wu, J. Song, J. Zhou, and H. Jiang, Chin. Phys. B 25, 117311 (2016), arXiv: 1711.10725.

6 E. J. Meier, F. A. An, A. Dauphin, M. Maffei, P. Massignan, T. L.
Hughes, and B. Gadway, Science 362, 929 (2018), arXiv: 1802.02109.

7 S. Stützer, Y. Plotnik, Y. Lumer, P. Titum, N. H. Lindner, M. Segev, M. C. Rechtsman, and A. Szameit, Nature 560, 461 (2018).

8 S. Yao, and Z. Wang, Phys. Rev. Lett. 121, 086803 (2018), arXiv: 1803.01876.

9 Z. Gong, Y. Ashida, K. Kawabata, K. Takasan, S. Higashikawa, and M. Ueda, Phys. Rev. X 8, 031079 (2018), arXiv: 1802.07964.

10 R. El-Ganainy, K. G. Makris, M. Khajavikhan, Z. H. Musslimani, S. Rotter, and D. N. Christodoulides, Nat. Phys. 14, 11 (2018).

11 D.-W. Zhang, L.-Z. Tang, L.-J. Lang, H. Yan, and S.-L. Zhu, Sci. China-Phys. Mech. Astron. 63, 267062 (2020).

12 X.-W. Luo, and C. Zhang, arXiv: 1912.10652. 